\title{
Öffentliche Finanzen im Spiegel der Verfassungsrechtsprechung
}

von Werner Heun

Das Grundgesetz regelt im Gegensatz zu anderen Verfassungen die öffentlichen Finanzen besonders eingehend. Die Verfassungsrechtsprechung hat dennoch erst relativ spät die Haushalts- und Finanzverfassung zum Gegenstand ihrer Entscheidungen gemacht, darunter vor allem den eigentlichen horizontalen Finanzausgleich und die Staatsverschuldung. Gleichwohl ist die Kontrollintensität ebenso wie die Wirkung der Verfassungsrechtsprechung anders als im Bereich des Steuerrechts und seiner Kontrolle am Maßstab des Gleichheitssatzes eher gering.

The Basic Law regulates the public finances with great detail compared to other constitutions. Nevertheless constitutional jurisprudence has dealt with the fiscal constitution only relatively late. Especially the decisions on fiscal equalization and public debt are important. The intensity of judicial review and its effects in this field of law are smaller than the judicial review of tax law where the equal protection clause is the standard of review.

\section{Einleitung}

Die öffentlichen Finanzen bilden das Rückgrat des modernen Staates, die „Nerven der Republik“, wie schon Bodin bemerkt hat. ${ }^{1}$ Sie waren der Ausgangspunkt für die Entstehung des ständestaatlichen Finanzstaats ${ }^{2}$ und Hauptbeweggrund für die Schaffung demokratischer Parlamente im Konstitutionalismus. ${ }^{3}$ Gleichwohl findet dieser Tatbestand in den modernen Verfassungen häufig nur begrenzt Ausdruck. Lediglich Kernelemente des Haushaltskreislaufs und in Bundesstaaten allenfalls rudimentäre Grundlagen der föderativen Finanzbeziehungen werden verfassungsrechtlich geregelt. Das Grundgesetz ist auch in diesem Fall eine Ausnahmeerscheinung, da es sowohl den Budgetzyklus als auch die föderative Finanzverfassung im Vergleich zu anderen Verfassungsordnungen mit besonderer Detailfreude in Art. 104a bis 115 GG normiert hat. Dennoch haben sich diese

\footnotetext{
Bodin, J.: Les Six Livres de la République, Paris, 1583, L. VI, c. (855).

Heun, W: Staatshaushalt und Staatsleitung, Baden-Baden, 1989, $40 \mathrm{ff}$.

Heun, $W$.: a.a.O., $52 \mathrm{ff}$.
} 
Bereiche des Verfassungsrechts lange weitgehend dem Zugriff der Verfassungsrechtsprechung entzogen. Sieht man von vereinzelten Entscheidungen des Bundesverfassungsgerichts ab, die Randfragen betrafen, ${ }^{4}$ setzen die substantiellen und zentralen Urteile erst Mitte der achtziger Jahre des vorigen Jahrhunderts ein. Zuerst wird ab 1986 der bundesstaatliche Finanzausgleich zum Gegenstand einer Reihe von grundlegenden Entscheidungen, ${ }^{5}$ ab 1989 folgen dann die beiden Urteile zur Staatsverschuldung. ${ }^{6}$ Gleichwohl bleiben bis heute noch wesentliche Bereiche der Haushalts- und Finanzverfassung von der Verfassungsrechtsprechung ausgespart. Demgegenüber wurden die Besteuerung der Bürger vor allem unter dem Gesichtspunkt des Grundsatzes der Gleichmäßigkeit der Besteuerung (Art. $3 \mathrm{GG})^{7}$ und die Kompetenzen des Staates zur Abgabenerhebung, insbesondere zur Auferlegung von Sonderabgaben, ${ }^{8}$ von Anfang an einer intensiven Kontrolle durch das Bundesverfassungsgericht unterworfen.

In dieser markanten Differenz zwischen der budgetären und föderativen Ausgabenwirtschaft einerseits und der Erhebung der Abgaben vom Bürger durch den Staat andererseits spiegeln sich die materiellen und prozessualen Unterschiede zwischen staatsinternen und das Außenverhältnis zwischen Staat und Bürger regelnden Normenkomplexen wider. Letzteres ist materiell durch die individuellen Grundrechte und prozessual durch die jedem Bürger offenstehende Verfassungsbeschwerde, ersteres durch das Staatsorganisationsrecht und die Verfahren des Organstreits und der föderativen Streitigkeiten geprägt. Die Verfahrensbeteiligten dieser Verfahren, die Verfassungsorgane zum einen sowie Bund und Länder zum anderen, gehen politisch viel selektiver vor. Zugleich sind die Steuerungs- und Einflussmöglichkeiten der Verfassungsrechtsprechung wesentlich schwächer. Im Bereich des Grundrechtsschutzes ist das Bundesverfassungsgericht deutlich durchsetzungswilliger und -fähiger. Deswegen vermittelt die Verfassungsrechtsprechung im Bereich der Ausgabenwirtschaft, auf die im Folgenden vornehmlich eingegangen werden soll, ein viel unvollständigeres Bild des Gesamtspektrums als im Bereich des abgabenrelevanten Staat-BürgerVerhältnisses.

4 Z.B. BVerfGE 20, 56 - Parteifinanzierung, 45, 1 - Haushaltsüberschreitung, sowie 39, 96 - Städtebauförderungsgesetz und 41, 291 - Strukturförderung.

5 BVerfGE 72, 330 Finanzausgleich I; 86, 148 Finanzausgleich II; 101, 158 Finanzausgleich III; 116, 327 - Berliner Haushalt.

6 BVerfGE 79, 311 - Staatsverschuldung I; 119, 96 Staatsverschuldung II.

7 Vgl. im Einzelnen Heun, W.: in: H. Dreier (Hrsg.), GG-Kommentar, Bd. I, 3. Aufl. 2013, Art. 3, Rn. 75 ff.; grundlegend Birk, D.: Das Leistungsfähigkeitsprinzip als Maßstab der Steuernormen, 1983, 21 ff., $156 \mathrm{ff}$.

8 Siehe dazu im Folgenden II, 3. 


\section{Die föderativen Finanzbeziehungen}

\section{Der Stufenbau der Finanzverfassung}

Die bundesstaatliche Finanzverfassung des Grundgesetzes ${ }^{9}$ zeichnet sich durch einen komplizierten Stufenbau aus. Die Regeln der Finanzverfassung, die in Art. 104a bis 109 GG normiert sind, legen die gegebene verfassungsrechtliche Aufgabenverteilung zugrunde, die auch einen sachlichen Vorrang genießt, ohne die Finanzverfassung zur Folgeverfassung zu degradieren. ${ }^{10}$ Die Finanzverfassung beginnt daher mit der Festlegung der Verantwortung für die anfallenden Kosten in Art. 104a GG. Daran schließen sich die verschiedenen Elemente der Einnahmenverteilung an, die sich in drei Kategorien einteilen lassen. Erstens müssen die Gesetzgebungskompetenzen für die zu erhebenden Abgaben, die sogenannte Objekthoheit, auf Bund und Länder verteilt werden. Art. 105 GG weist die entsprechenden Kompetenzen zu, beschränkt sich aber auf die Gesetzgebungsbefugnis für die Steuern. Dabei setzt Art. 105 GG den Steuerbegriff voraus, der verfassungsrechtlich in Abgrenzung $\mathrm{zu}$ den anderen Abgabenarten gewonnen wird. ${ }^{11}$ Durch diese Beschränkung auf die Regelung der Steuern und ihre faktische Bedeutung wird die Bundesrepublik verfassungsrechtlich nicht auf den Steuerstaat festgelegt, ${ }^{12}$ auch wenn das Bundesverfassungsgericht sich bei einer Gelegenheit in diese Richtung geäußert hat. ${ }^{13}$ Zweitens müssen die Erträge aus den Abgaben auf die beiden föderativen Ebenen verteilt werden. Die Regelung dieser Ertragshoheit findet sich in Art. 106 GG. Schließlich müssen drittens die aufgrund der Ertragshoheit auf die Länder entfallenden Einnahmen zwischen den Ländern verteilt (Art. 107 I GG) sowie gegebenenfalls Korrekturen vorgenommen werden, für die das Grundgesetz verschiedene Instrumente vorsieht (Art. 107 I 4 2. HS, 107 II GG).

Als letztes Element der Kompetenzverteilung regelt die Verfassung in Art. 108 GG schließlich noch die Zuordnung der Verwaltungs- und der Gerichtshoheit für Steuern.

9 Eingehende Darstellungen: Korioth, St:: Der Finanzausgleich zwischen Bund und Ländern, 1997; Häde, U.: Finanzausgleich, 1996.

10 So aber Kirchhof, F.: Grundsätze der Finanzverfassung des vereinten Deutschlands, VVDStRL 52 (1993), 71 - 110 (80); dagegen Heun, W.: in: H. Dreier (Hrsg.), GG-Kommentar, Bd. III, 2. Aufl. 2008, Vorb. Art. 104a-115, Rn. 20.

11 Vgl. schon BVerfGE 3, 407 (435); Heun, in: Dreier, Bd. III, aaO., Art. 105, Rn. 12.

12 Heun, W.: Die Entwicklung des Steuerstaatskonzepts in theoretischer und tatsächlicher Hinsicht, in: U. Sacksofsky/ J. Wieland (Hrsg.), Vom Steuerstaat zum Gebührenstaat, 2000, 10-21 (19 ff.); zuletzt Thiele, A.: Finanzaufsicht, 2014, 439 f. mwN.

13 BVerfGE 78, 249 (266 f.). 
Das Element, das in der stufenweisen Verteilung von Kompetenzen und Einnahmen am prekärsten ist, ist die Korrektur der primären Einnahmenverteilung durch den Finanzausgleich, der dementsprechend das eigentliche Zentrum der Entscheidungen des Bundesverfassungsgerichts bildet. Daneben sind lediglich die Gesetzgebungskompetenzen für die Sonderabgaben, die nicht von den Steuergesetzgebungskompetenzen gedeckt sind und $\mathrm{zu}$ den Steuern in Konkurrenz treten, häufiger Gegenstand der Rechtsprechung geworden. Die anderen Normen treten allenfalls am Rande in den Blick.

\section{Ausgabenverantwortung}

Der 1969 eingefügte Art. 104a Abs. 1 GG normiert die allgemeine Lastenverteilungsregel nach der die Ausgabenverantwortung der Wahrnehmung der zugewiesenen Aufgaben folgt. Dieses Konnexitätsprinzip versteht unter Aufgabenverantwortung die verfassungsrechtlich zugewiesene Verwaltungszuständigkeit. Schon vor 1969 hat das Bundesverfassungsgericht diesen Grundsatz in diesem Verständnis als ungeschriebenes Prinzip anerkannt. ${ }^{14}$ Ansonsten hat das Bundesverfassungsgericht sich in diesem Bereich schon relativ früh lediglich zu den früher in Art. 104a Abs. 4 GG, heute in Art. 104b GG geregelten Finanzhilfen als Modifikation der Lastenverteilungsregel geäußert. Die Finanzhilfen werden aus dem Blickwinkel der bundesstaatlichen Kompetenzordnung eng begrenzt, da sie „die Gefahr von Abhängigkeiten der Länder vom Bund“ schaffen und „damit die verfassungsrechtlich garantierte Eigenständigkeit der Länder“ gefährden. ${ }^{15}$

Die Zuständigkeit erschöpft sich in der Gewährung der Finanzhilfen unter den dort genannten Voraussetzungen und darf - anders als etwa in den USA - nicht mit zusätzlichen Bedingungen verknüpft werden. Diese restriktive Haltung des Gerichts hat gleichwohl nicht verhindert, dass sich außerhalb der Zuständigkeitsverteilung des Art. 104a GG der Bund durch den goldenen Zügel der Finanzhilfen auch Einfluss auf die Entscheidungen der Länder verschafft hat. Das insoweit in Art. 104b GG verankerte striktere Kooperationsverbot der letzten Föderalismusreform, das dem teilweise entgegensteht, soll daher auch wieder fallen. Auch schon vor der Verankerung der Gemeinschaftsaufgaben in der Finanzreform hatte sich eine weitgehend extrakonstitutionelle Fondswirtschaft entwickelt, ${ }^{16}$ die nachträglich legalisiert wurde. Dies ist einer der Bereiche, in dem die Macht des

16 Müller-Volbehr, J.: Fonds- und Investitionshilfekompetenz des Bundes, 1975, 36 ff. 
Bundesverfassungsgerichts begrenzt ist, da keiner der Beteiligten ein Interesse an einer verfassungsgerichtlichen Konfliktentscheidung hat.

\section{Abgabenkompetenzen}

Im Bereich der eigentlichen Steuergesetzgebungskompetenzen hat das Bundesverfassungsgericht kaum Gelegenheit für eigene Entscheidungen erhalten. Abgesehen von Urteilen zu Zöllen und Finanzmonopolen ist Art. 105 GG nur insoweit Anlass für Streitigkeiten geworden, als es um die Sperrwirkung von Steuergesetzen des Bundes für solche der Länder und Gemeinden im Fall der konkurrierenden Gesetzgebungskompetenz ging, die an der Gleichartigkeit als Voraussetzung einer Kollision ansetzt. Sachlich werden dadurch aber auf der Länderebene lediglich Steuern von marginaler Bedeutung und vor allem örtliche Steuern berührt. Gravierenden Einfluss auf die öffentlichen Finanzen hatten diese Entscheidungen daher nicht.

Wesentlich bedeutsamer ist insofern die Verfassungsrechtsprechung für die Erhebung von Sonderabgaben außerhalb der Steuergesetzgebungskompetenzen geworden. Ihre Bedeutung für die öffentlichen Finanzen liegt zunächst einmal darin, dass die Sonderabgaben Ausdruck des Grundprinzips sind, dass die Einführung neuer Abgabenformen verfassungsrechtlich nicht grundsätzlich ausgeschlossen ist. ${ }^{17}$ Das ist bei der UMTS-Versteigerung vielleicht am deutlichsten demonstriert worden. ${ }^{18}$ Daraus folgt nicht, dass der Gesetzgeber sein Abgabenerfindungsrecht völlig frei ausüben könnte. Vor allem die außerhalb des Systems der klassischen Abgaben (Steuern, Gebühren, Beiträge, Sozialversicherungsbeiträge) erhobenen Sonderabgaben sind verfassungsrechtlich problematisch, weil sie vielfach nicht im allgemeinen Haushalt veranschlagt werden, sie der finanzverfassungsrechtlichen Kompetenz- und Einnahmenverteilung entzogen sind und für den Bürger Sonderlasten begründen. ${ }^{19}$ Deshalb sind die Sonderabgaben allerdings noch nicht von vornherein unzulässig, sie bedürfen jedoch exakter und präziser Abgrenzung gegenüber anderen Abgaben und zudem vor allem einer spezifischen besonderen Rechtfertigung, ${ }^{20}$ die sie als eine Sonderform der Kausalabgaben qualifiziert. Diese Rechtfertigung kann in der Antriebs- und Ausgleichsfunktion, wie bei bestimmten Umweltabgaben, in der Abschöpfung des Vorteils einer staatlichen Handlung oder Leistung, wie bei den Konzessionsab-

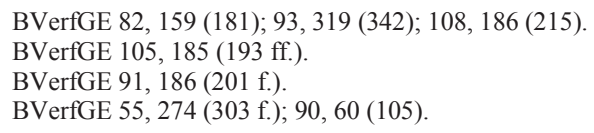


gaben, oder in der besonderen gruppennützigen Verwendung der Einnahmen, wie beispielsweise bei der Berufsbildungsabgabe, liegen. ${ }^{21}$ Jedenfalls zieht die Verfassungsrechtsprechung dem grundsätzlich anerkannten Abgabenerfindungsrecht damit durchaus deutliche Grenzen.

\section{Ertragshoheit und horizontaler Finanzausgleich}

Die Zuordnung der Ertragshoheit der einzelnen Steuern zu den verschiedenen Ebenen in Art. 106 GG ist im Wesentlichen unproblematisch und daher auch nicht Gegenstand von verfassungsgerichtlichen Entscheidungen geworden. Das Gegenteil gilt für die endgültige Verteilung der Einnahmen auf die Länder, die letztlich über deren tatsächliche Finanzausstattung entscheidet. Deswegen ist es schon beinahe erstaunlich, dass erst 1986 die erste in einer Reihe von Entscheidungen zum Kern des horizontalen Finanzausgleichs ergangen ist. ${ }^{22}$ Das Gericht geht bei seiner Analyse und Bewertung ganz von allgemeinen juristischen Prinzipien aus und lässt ökonomische Gesichtspunkte weitgehend außer Betracht. Nach Auffassung des Bundesverfassungsgerichts sind im Rahmen des horizontalen Finanzausgleichs die bundesstaatlich gewollte Autonomie und Eigenständigkeit der Länder einerseits und die bundesstaatliche Solidarität und Mitverantwortung andererseits, die durch das Resultat der Einheitlichkeit der Lebensverhältnisse noch sozialstaatlich unterfangen wird, ${ }^{23}$ die leitenden verfassungsrechtlichen Grundsätze, die einem Kompromiss zuzuführen sind.

Die Grundprinzipien geben freilich lediglich hochabstrakte Ziele vor und begründen ebenso abstrakte Rechte und Pflichten, die ohne nähere Konkretisierung keine hinreichend präzisen Beurteilungs- und Bewertungsmaßstäbe ergeben. Die Möglichkeit, bei der Konkretisierung auf ökonomische Kriterien und Maßstäbe, insbesondere auf externe Effekte und die fiskalische Gleichheit zurückzugreifen, hat das Bundesverfassungsgericht nicht genutzt. Schon angesichts der Offenheit der Normen und Maßstäbe kam das Bundesverfassungsgericht nicht umhin, dem Gesetzgeber für die Regelungen des Finanzausgleichs einen (weiten) Gestaltungsspielraum zuzuerkennen. ${ }^{24}$ Es hat aber versucht, diesem Gestaltungsspielraum äußerste Grenzen zu ziehen - dieser Anspruch wird dadurch untermauert, dass die finanzverfassungsrechtlichen Normen nicht als Recht minderer Gel-

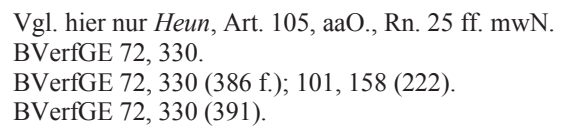


tungskraft, als bloßes soft law, qualifiziert werden dürfen, ${ }^{25}$ sondern den Beteiligten nur Entscheidungs- und Beurteilungsspielräume eingeräumt werden. Indes erwies es sich als schwierig, derartige Grenzen näher zu bestimmen. Als sachliche Schranke hat das Bundesverfassungsgericht daher letztlich nur das Verbot der völligen Nivellierung und Egalisierung der Länderfinanzen ${ }^{26}$ sowie das Verbot der Vermeidung der Finanzkraftreihenfolge ${ }^{27}$ aufgestellt. Diese Prinzipien und Grenzen sind plausibel und einleuchtend, bewirken aber praktisch nichts. Das extrem hohe Ausgleichsniveau und der faktische Vorrang des Distributionsziels der fiskalischen Gleichheit bei der Ausgestaltung des Finanzausgleichs, die freilich in der differenzierten Stufenfolge des Finanzausgleichs verfassungs- und einfachrechtlich angelegt sind, sind dadurch in keiner Weise beschnitten oder zurückgedrängt worden. Vermutlich ist das Verfassungsrecht damit auch angesichts der starken unitarischen politischen Kräfte überfordert.

Konkrete Folgen für die Ausgestaltung des Finanzausgleichs hat das Bundesverfassungsgericht durch andere Gesichtspunkte erzielt. Während auch bei der Zerlegung, d.h. der Verteilung der Einnahmen aus einem Steuertatbestand (Einkommensteuer, Körperschaftsteuer) auf die Länder, lediglich die Ziele der Erfassung der wirklichen Steuerkraft und des Abbaus einnahmebedingter Verzerrungen gelten und der Gesetzgeber bei der Bestimmung von Art und Umfang der Zerlegung über einen Gestaltungsspielraum verfügt, ${ }^{28}$ hat das Bundesverfassungsgericht immerhin bei der Bestimmung der Finanzkraft erreicht, dass alle ausgleichsrelevanten Einnahmen in die Berechnung einbezogen werden müssen und der Begriff nicht auf die Steuerkraft reduziert werden darf. ${ }^{29}$ Nichtsteuerliche Einnahmen dürfen folglich bei der Ermittlung der Finanzkraft nur unberücksichtigt bleiben, wenn sie ihrem Volumen nach nicht ausgleichsrelevant sind, wenn sie in allen Ländern verhältnismäßig anfallen oder wenn der Aufwand für die Ermittlung zu dem möglichen Ausgleichseffekt außer Verhältnis steht. ${ }^{30}$ Die bergrechtliche Förderabgabe war daher beispielsweise zu Lasten Niedersachsens zu berücksichtigen. ${ }^{31}$

Im Hinblick auf die Vorschrift des Art. 107 Abs. 2, Satz 1 2. Hs. GG, wonach die Finanzkraft und der Finanzbedarf der Kommunen bei der Ermittlung der Finanz-

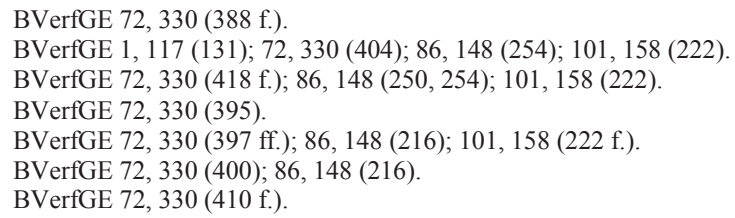


kraft „Zu berücksichtigen“ sind, hat das Gericht allerdings dem Gesetzgeber Spielräume eröffnet. Auch unter dem Aspekt der Garantie kommunaler Autonomie gemäß Art. 28 Abs. 2 GG können insoweit die kommunalen Einnahmen zur Hälfte außer Ansatz gelassen werden. ${ }^{32}$ Der Effekt für die Finanzausstattung der Länder ist erheblich, die weitgehende rechnerische Nivellierung der so berechneten Finanzkraft wird dadurch stark relativiert und lässt diesen Ausgleichsmechanismus im Ergebnis auch akzeptabel erscheinen.

Da der Finanzbedarf bei dem Finanzausgleich offiziell ausgeblendet bleibt, allerdings durch den Einwohnermaßstab sachlich doch, wenngleich relativ abstrakt, berücksichtigt wird, hängt alles von dem Verständnis des ,,angemessenen Ausgleichs" in Art. 107 Abs. 2 Satz 1 GG ab. Die Leitformel des Bundesverfassungsgerichts für die inhaltliche Ausgestaltung greift auf die allgemeinen Prinzipien zurück; danach habe der Ausgleich „die richtige Mitte zu finden zwischen der Selbständigkeit, Eigenverantwortlichkeit und Bewahrung der Individualität der Länder auf der einen und der solidargemeinschaftlichen Mitverantwortung für die Existenz und Eigenständigkeit der Bundesgenossen auf der anderen Seite “. ${ }^{33}$ Diese letztlich nur als Ausprägung des Bundesstaatsprinzips zu verstehende Formel trägt jedenfalls innerhalb der Grenzen des Nivellierungsverbots praktisch nichts aus und muss die Konkretisierung der gesetzgeberischen Gestaltung überlassen.

Kaum geringer ist der Entscheidungsspielraum des Gesetzgebers bei den sogenannten Ergänzungszuweisungen des Bundes gemäß Art. 107 Abs. 2 Satz 3 GG, die zum Ausgleich besonderer Finanzschwäche (Fehlbetragszuweisungen) oder übermäßiger Belastungen (Sonderbedarfszuweisungen) dienen sollen. Diese subsidiären Ergänzungszuweisungen sind fakultativ und stehen im Ermessen des Gesetzgebers, können sich aber zu einer Rechtspflicht verdichten. ${ }^{34}$ Gerade anfangs war das Bundesverfassungsgericht hier großzügig und hat höchst problematische Ergänzungszuweisungen, wie etwa die angeblich erhöhten Kosten politischer Führung, ${ }^{35}$ akzeptiert.

Noch zweifelhafter ist die Rechtsprechung des Bundesverfassungsgerichts jedenfalls anfänglich für den Fall der sogenannten Haushaltsnotlagen. In seinem zweiten Finanzausgleichsurteil hat es dem Gesetzgeber sogar die Verpflichtung auferlegt, im Fall einer extremen Haushaltsnotlage den betreffenden Ländern

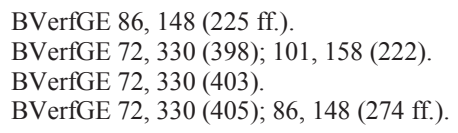


Sonderbedarfszuweisungen zur Behebung dieser Notlage zukommen zu lassen, wobei Bund und Länder gemeinsam die finanziellen Lasten zu tragen haben. ${ }^{36}$ Da derartige Haushaltsnotlagen zumindest zu einem erheblichen Teil auch auf politisch-autonomen Entscheidungen beruhen, setzt diese Judikatur deutliche Fehlanreize und ist höchst fragwürdig, auch wenn es zulässig ist, die Zuweisungen mit der Auflage eines Haushaltssanierungsprogramms zu verbinden. Als das Land Berlin prompt einen entsprechenden Anspruch geltend machte, hat das Gericht jedoch mit einer gewissen Volte eine restriktive Haltung eingenommen und durch seine grundsätzlichen verfassungsrechtlichen Bedenken seine eigene frühere Position unterminiert. ${ }^{37}$

\section{Das Konzept des Maßstäbegesetzes}

Angesichts der geringen Steuerungskraft materieller Prinzipien ist das Bundesverfassungsgericht auf die Idee verfallen, durch die Vorgabe eines gesonderten Maßstäbegesetzes den Entscheidungsprozess des Länderfinanzsaugleichs verfahrensrechtlich zu rationalisieren. ${ }^{38}$ Danach sollen die abstrakten Verteilungskriterien in einem von den konkreten Verteilungsentscheidungen gelösten Maßstäbegesetz hinter einem „Schleier des Nichtwissens“ festgelegt werden, das dann die konkrete Verteilung im zweiten Ausgleichsgesetz verbindlich determinieren soll. Dieses Konzept ist insofern naiv, als die Entscheidung über die abstrakten Kriterien selbstverständlich von den Beteiligten in Kenntnis und im Hinblick auf die daraus resultierenden finanziellen Auswirkungen getroffen wird. Dogmatisch ist die Konstruktion einer im Rang zwischen Verfassung und einfachem Gesetz angesiedelten neuen Gesetzeskategorie mit der Normenordnung des Grundgesetzes kaum vereinbar. Das Diktum des Bundesverfassungsgerichts setzt die lexposterior Regel nicht außer Kraft. Es verwundert daher nicht, dass der Gesetzgeber sich im Maßstäbegesetz faktisch auf die Wiedergabe der verfassungsrechtlichen grundlegenden Vorgaben beschränkt hat und damit die Konstruktion des Maßstäbegesetzes leerlaufen lässt. 


\section{Haushaltswesen und Staatsverschuldung}

\section{Der Haushaltskreislauf}

Die Haushaltswirtschaft spielt sich in einem festen Zyklus aufeinanderfolgender Stufen des Entscheidungsprozesses ab: Planung und Aufstellung des Haushalts durch die Exekutive, Beratung und Beschlussfassung im Parlament, Vollzug durch die Exekutive unter gelegentlicher Beteiligung des Parlaments, Finanzkontrolle durch Rechnungshof und Parlament mit abschließender Entlastung. ${ }^{39}$ Der Prozess zeichnet sich durch eine enge Kooperation zwischen Parlament und Exekutive aus, die weitgehend nur bei einem entsprechenden gegenseitigen Vertrauensverhältnis funktioniert. Das lässt kaum Raum für Konfliktentscheidungen durch ein Verfassungsgericht, sodass schon aus diesem Grund nur ganz wenige Judikate aus diesem Bereich vorliegen. Einzelne Vorschriften des Grundgesetzes aus den Art. 110 bis 114 GG sind zudem funktionslos, wie Art. 113 GG, der der Regierung ein Einspruchsrecht gegen ausgabenerhöhende und einnahmenmindernde Gesetze einräumt. Rechnungsprüfung, parlamentarische Finanzkontrolle und Entlastung nach Art. 114 GG sind im Wesentlichen informeller Natur und können deshalb kaum Anlass zu einem Verfassungsstreit sein. Im Grunde hat das Bundesverfassungsgericht deshalb nur in drei Fällen substanziell zu Fragen des Haushaltsverfassungsrechts Stellung genommen. Dazu treten schließlich noch zwei Entscheidungen zur Staatsverschuldung, die unabhängig vom Budgetzyklus sind.

Recht frühzeitig, nämlich 1966, ergriff das Bundesverfassungsgericht anlässlich des Verfahrens über die Parteienfinanzierung die Gelegenheit, den klassischen Streit über die Rechtsnatur und die Rechtswirkungen des Haushaltsplans zu entscheiden. Es konstatierte gegen die Auffassung von Paul Laband, ${ }^{40}$ das Haushaltsgesetz gemäß Art. 110 GG stelle ,nicht lediglich ein im Haushaltsplan enthaltendes rechtsindifferentes Zahlenwerk fest und schafft nicht nur einen tatsächlichen Zustand“, sondern bewilligt die im Budget ausgeworfenen Mittel und ermächtigt die Exekutive überhaupt erst zur Verausgabung. ${ }^{41}$ Diese Ermächtigung schafft zudem Befugnisse und Verantwortlichkeiten im organschaftlichen Rechtskreis und ist ein aus sich heraus verständlicher Rechtssatz, der auf seine förmliche und sachliche Vereinbarkeit mit dem Grundgesetz überprüft werden

39 Eingehend dazu Heun, Staatshaushalt, aaO., $291 \mathrm{ff}$.

40 Laband, P.: Das Staatsrecht des Deutschen Reiches, Bd. II 2. Abt. 1882, 339 ff.; zur damaligen Diskussion Heun, Staatshaushalt, aaO., 79 ff.; Friauf, K.-H.: Der Staatshaushaltsplan im Spannungsfeld zwischen Parlament und Regierung, 1968, $249 \mathrm{ff}$.

41 BVerfGE 20, 56 (91); vgl. Heun, Staatshaushalt, aaO., $151 \mathrm{ff}$. 
und gültig oder nichtig sein kann. ${ }^{42}$ Das Budget war damit auch grundsätzlich der verfassungsgerichtlichen Kontrolle unterworfen.

Gut zehn Jahre später erreichte der nächste Streit das Bundesverfassungsgericht, der die Bewilligung des Bundesfinanzministers von über- und außerplanmäßigen Ausgaben gemäß Art. 112 GG betraf. Das Verfahren eröffnete dem Gericht die Möglichkeit sich zum Nothaushaltsrecht gemäß Art. 111 GG und zum Notbewilligungsrecht gemäß Art. 112 GG zugleich zu äußern, da die Bewilligungen noch im etatlosen Zustand erfolgten. Zu Recht hob das Gericht den Ausnahmecharakter des Nothaushaltsrechts der Exekutive im etatlosen Zustand hervor und kritisierte die als verfassungswidrig qualifizierte langjährige Praxis, dass der Haushalt meist mit halbjähriger Verspätung erlassen wurde. Die faktische Fortschreibung des jeweils bisherigen Haushaltsplans verletze das Etatbewilligungsrecht des Parlaments. ${ }^{43}$ Die Kritik war heilsam, da in der Folgezeit die Haushaltsgesetze lange rechtzeitig vor Beginn des Haushaltsjahres verabschiedet wurden. Erst in jüngster Zeit hat die Disziplin nachgelassen. Außerdem wurde die extensive Handhabung des Notbewilligungsrechts des Bundesfinanzministers, die auch durch die Etatlosigkeit befördert wurde, durch eine restriktivere Interpretation des Art. 112 GG zurückgeschnitten. ${ }^{44}$ Insgesamt wurde dadurch das ohnehin bestehende Übergewicht der Exekutive zugunsten des Parlaments etwas abgeschwächt. Wiederum zehn Jahre später wurde akzeptiert, dass die Bewilligung und Kontrolle der geheimen Wirtschaftspläne der Nachrichtendienste einem kleinen Gremium anvertraut wurden, in dem nicht alle Fraktionen vertreten waren. ${ }^{45}$ Das Gericht betonte hier erneut die Bedeutung des Haushaltsplans als staatsleitender Hoheitsakt in Gesetzesform und des Budgetrechts als wesentlichem Instrument der parlamentarischen Regierungskontrolle, ${ }^{46}$ sah aber in der weitgehenden Ausgliederung der Wirtschaftspläne keine Verletzung haushaltsrechtlicher Grundsätze und akzeptierte gegen den berechtigten Protest der Minderheit ${ }^{47}$ den Ausschluss einzelner Fraktionen mit dem Gedanken des Geheimschutzes und der Notwendigkeit des Vertrauens der Mehrheit im Parlament. $^{48}$

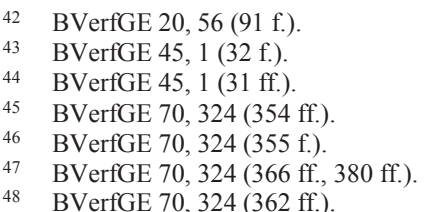




\section{Das Problem der Staatsverschuldung}

Die Staatsverschuldung verharrte in der Bundesrepublik nach der Entschuldung im Gefolge des Zweiten Weltkriegs lange auf niedrigem Niveau. Das änderte sich erst im Laufe der ersten wirtschaftlichen Krisen der 70er Jahre. Zu diesem Zeitpunkt war die Regelung der Kreditaufnahme im Zuge der Haushaltsreform 1969 bereits grundlegend unter dem Eindruck des Keynesianismus umgestaltet worden. ${ }^{49}$ Gleichwohl dauerte es noch bis 1989, bis das Bundesverfassungsgericht erstmals eine Entscheidung über die Zulässigkeit der Kreditaufnahme - im lange zurückliegenden Jahr 1981 - traf. ${ }^{50}$ Die verfassungsrechtliche Beurteilung stand vor interpretatorischen und sachlichen Schwierigkeiten unterschiedlicher Art. Geringe Probleme warf die Regelgrenze der Kreditaufnahme auf, die durch die Höhe der Ausgaben für Investitionen definiert war. Der unbestimmte Rechtsbegriff der Investitionen war im Grundgesetz zwar nicht definiert, galt aber zu Recht als in vollem Umfang justitiabel. Die Abgrenzung der Investitionen mag im Einzelfall schwierig sein, ist aber juristisch nicht unlösbar. Das Bundesverfassungsgericht orientierte sich daher an der weithin konsentierten Staatspraxis. ${ }^{51}$

Eine weitaus größere Herausforderung stellte die Zulässigkeit der Überschreitung der Regelgrenze zur Abwehr einer Störung des gesamtwirtschaftlichen Gleichgewichts dar. Zwar konnte das Gericht bei der Interpretation des gesamtwirtschaftlichen Gleichgewichts auf dessen Konkretisierung durch das sogenannte „Magische Viereck“" in $\S 1$ StabG zurückgreifen, ohne dass dadurch die hohe Komplexität der Beurteilung reduziert worden wäre. Mit anderen Worten: Die Beurteilung, ob eine Störung des gesamtwirtschaftlichen Gleichgewichts vorlag und welche Maßnahmen zur Abwehr der Störung geeignet waren, war dem Bundesverfassungsgericht letztlich entzogen. Dem versuchte das Gericht durch die Kombination von drei Argumenten zu begegnen. Erstens wurde dem Haushaltsgesetzgeber ein Einschätzungs- und Beurteilungsspielraum eingeräumt. ${ }^{52}$ Zweitens wurde die Überschreitung als strikte Ausnahmeregelung qualifiziert. ${ }^{53}$ Drittens - und das war der wichtigste Schritt wurde die Offenheit des materiellen Prüfungsmaßstabs durch die formellverfahrensmäßigen Anforderungen einer Darlegungslast für die Erfüllung der Voraussetzungen zu kompensieren versucht. ${ }^{54}$ Diese Darlegungslast findet freilich im Grundgesetz keinerlei Anhaltspunkte, verkennt die Pflichten des Gesetzgebers, der

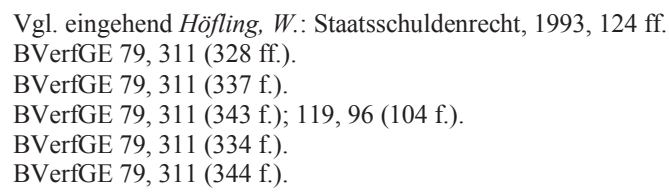


gerade keine Begründung schuldet, und versagt schließlich auch als Kontrollinstrument, da das Gericht dadurch kaum in die Lage versetzt wird, die Störung und ihre Abwehr ernsthaft besser zu beurteilen. Praktisch musste das Gericht daher auch in dem zweiten Verfahren die Überschreitung der Regelgrenze akzeptieren, ${ }^{55}$ wenngleich unter Protest zweier dissentierender Richter, ${ }^{56}$ deren Einschätzung der Situation aber kaum fachkundiger war. Dieses letztlich nicht lösbare Dilemma führte auch zu der Forderung nach einer Revision der Norm, ${ }^{57}$ der der verfassungsändernde Gesetzgeber nach der Finanzkrise 2008 mit dem zweifelhaften Ergebnis der sogenannten Schuldenbremse folgte, die ein prinzipielles Verbot der Kreditaufnahme mit nur eng begrenzten Ausnahmen vorsieht. ${ }^{58}$ Auch die geringe Befolgung der Maßgaben des Bundesverfassungsgerichts durch den Gesetzgeber demonstriert, dass die Steuerungskraft verfassungsgerichtlicher Entscheidungen im Bereich staatlicher Verschuldung in besonders hohem Maß an ihre Grenzen stößt.

\section{Resümee}

Die Rechtsprechung des Bundesverfassungsgerichts zu den öffentlichen Finanzen hinterlässt einen etwas zwiespältigen Eindruck. Das Gericht betont den verbindlichen Charakter des Haushalts- und Finanzverfassungsrechts und hat in einzelnen Fällen, wie beim Notbewilligungsrecht, wirkungsvolle Anstöße gegeben.

Beim Finanzausgleich stößt das Gericht auch angesichts der durch die Verfassung eröffneten Spielräume an die Grenzen der Steuerungsfähigkeit des Finanzrechts. Trotz hohen Aufwands der Rechtsprechung ist die Wirkung gering. Im Bereich der Staatsschulden ist die Wirkungskraft der Entscheidungen noch geringer, die Judikate werden hier nicht nur unterlaufen, wie im Finanzausgleich, sondern teilweise beinahe offen ignoriert, zumal das Gericht in diesen Fällen mit großer Verspätung entscheidet und auch nur über eine laienhafte Sachkenntnis verfügt.

Insgesamt erweist sich die Haushalts- und Finanzverfassung daher zwar nicht als Arkanum des Staatsrechts, aber doch als ein der verfassungsgerichtlichen Kontrolle nur begrenzt zugänglicher Bereich.

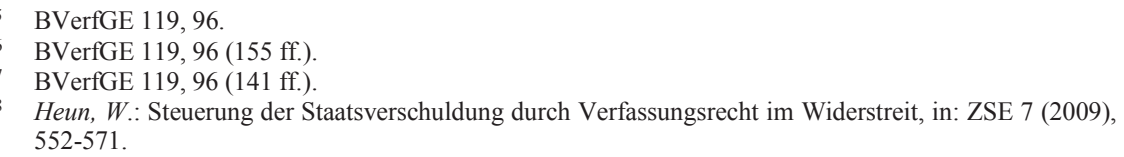

